Research Paper

\section{Analysis of Subsurface Scattering Based on Dipole Approximation}

\author{
Yasuhiro Mukaigawa, ${ }^{\dagger 1}$ KAZUYA SuzukI $^{\dagger 1}$ \\ and YASUSHI YAGI ${ }^{\dagger 1}$
}

The scattering effect of incident light, called subsurface scattering, occurs under the surface of translucent objects. In this paper, we present a method for analyzing the subsurface scattering from a single image taken in a known arbitrary illumination environment. In our method, diffuse subsurface reflectance in the subsurface scattering model can be linearly solved by quantizing the distances between each pair of surface points. Then, the dipole approximation is fit to the diffuse subsurface reflectance. By applying our method to real iman is fit to the diffureal images of translucent objects, we confirm that the parameters of subsurface scattering
can be computed for different materials.

\section{Introduction}

A variety of photometric analysis methods have been proposed to estimate the shading and reflection properties of images captured by a camera. Since it is assumed in most methods that the target scene consists of opaque materials, translucent objects cannot be dealt with. In translucent media, an incident ray scatters under the surface and reflects from different points. This reflection is called subsurface scattering. Although marble and skin are considered typical translucent objects, there are many more such objects, including fruit and vegetables ${ }^{1)}$. Some of the translucent objects in our everyday environment are shown in Fig. 1. However, many of the photometric analysis methods, such as shape-from-shading and photometric stereo, do not work well in our everyday environment. It is well known that the main reasons that these methods fail are non-Lambertian BRDFs (specular reflections), shadows, and inter-reflection. We believe that another reason is that these methods cannot deal with subsurface

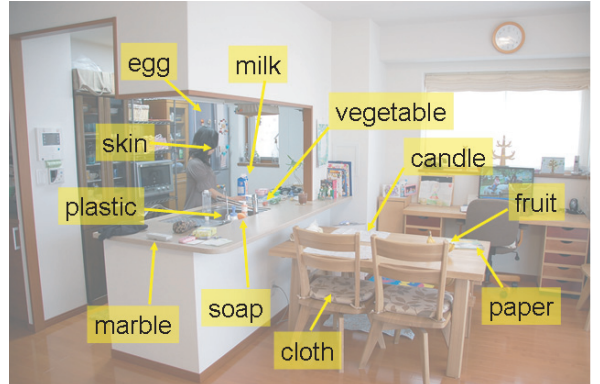

Fig. 1 Many translucent objects in our living environment.

scattering. Although the subsurface scattering comes close to Lambertian reflection under homogeneous illumination, fine-scale geometric features are blurred by the subsurface scattering. Hence, the analysis of subsurface scattering in translucent objects is very important.

In the computer graphics field, several algorithms for rendering translucent objects have been proposed. Hanrahan and Krueger ${ }^{2)}$ modeled reflection from layered surfaces such as skin and leaves. Stam ${ }^{3)}$ approximated the effects of multiple scattering in heterogeneous media by a diffusion process. Dorsey, et al. ${ }^{4)}$ proposed a Monte Carlo subsurface ray tracer to render stones. Pharr and Hanrahan ${ }^{5)}$ used scattering functions with Monte Carlo integration to render complex shapes. Jensen proposed photon mapping ${ }^{6)}$ which traces individual photons for simulating volumetric subsurface scattering. Later, Jensen, et al. proposed an analytic dipole approximation ${ }^{7}$ for multiple scattering in homogeneous materials based on a diffusion approximation, and extended it with a two-pass rendering technique ${ }^{8)}$. Donner ${ }^{9)}$ further extended this by a multipole diffusion approximation to render thin translucent slabs.

In the field of medical imaging, light scattering in the human body has been analyzed by diffuse optical tomography (DOT) ${ }^{10)}$. To reconstruct images, DOT requires modeling of the photon propagation in the scattering medium and data inversion. Nitta ${ }^{11)}$ proposed a weight function model for signal processing in DOT.

In the cosmetic field, skin translucency of facial images has been the focus of 
129 Analysis of Subsurface Scattering Based on Dipole Approximation

much research. Tsumura proposed a method to separate the hemoglobin and melanin components by independent component analysis ${ }^{12)}$, and estimated irradiance by the deconvolution of the point spread function of translucent media ${ }^{13)}$.

Translucent objects are, however, not the primary focus in the computer vision field. Most research focuses on analyzing diffuse and specular reflections and shadows on opaque objects. Only inter-reflection is analyzed as an effect of global illumination. Recently, various methods were proposed to measure subsurface scattering directly in translucent objects using special lighting devices such as a focused narrow beam using a lens ${ }^{7)}$, a sweeping laser beam ${ }^{14), 15)}$, a projector ${ }^{16), 17)}$, or a fiber optic spectrometer ${ }^{18)}$. Ghosh, et al. ${ }^{19)}$ proposed a practical system to measure and analyze layered facial reflectance within a few seconds. This system uses structured illumination and polarizers to estimate subsurface scattering parameters.

These methods can, however, analyze subsurface scattering only under strictly controlled illumination. In this paper, we propose a new method to analyze subsurface scattering using only a single image taken in a known arbitrary illumination environment. Such a problem setting is difficult to solve using the previous approaches. The challenge addressed in this study is that of analyzing subsurface scattering by inversely tracing the image rendering process.

Recently, Donner, et al. ${ }^{20}$ used an inverse rendering technique to obtain the parameters of subsurface scattering. In their model, human skin is modeled as layered heterogeneous material and subsurface scattering is controlled by five parameters. To estimate these parameters from a single, multi-spectral image, they utilized an inverse rendering approach. That is, starting from average skin parameters, a gradient descent optimization repeatedly renders the skin and finds the parameters that minimize the differences between the rendering and the acquired image.

Our approach is similar to this in that the parameters of subsurface scattering are estimated from a single image. However, our method divides the problem into a reflectance estimation and a parameter estimation. The former can be algebraically calculated by the least-squares method without any iterative calculation. The latter becomes a simple fitting problem of the dipole model to the estimated discrete reflectance. Consequently, we can eliminate the rendering process from the image analysis.

\section{Subsurface Scattering}

\subsection{Light Diffusion in Translucent Objects}

Most computer vision methods assume that the target scene consists of opaque objects. On the surface of an opaque object, an incident ray reflects directly at the incident point as shown in Fig. 2 (a). This reflection is expressed as a bidirectional reflectance distribution function (BRDF). The $\operatorname{BRDF} F\left(x, \omega_{i}, \omega_{o}\right)$ represents the ratio of outgoing radiance in the viewing direction $\omega_{o}$ to incident irradiance from a lighting direction $\omega_{i}$ at a surface point $x$.

On the other hand, an incident ray to a translucent object diffuses under the surface and reflects from different points. The incident and radiative points do not correspond as shown in Fig. 2 (b). Since strong scattering lights are observed around the incident point, the surface appears smooth because the irregularities of the surface become more difficult to see. This reflection is expressed as a bidirectional scattering surface reflectance distribution function (BSSRDF). The BSSRDF $S\left(x_{i}, \omega_{i}, x_{o}, \omega_{o}\right)$ represents the ratio of outgoing radiance in the viewing direction $\omega_{o}$ at $x_{o}$ to incident irradiance from a lighting direction $\omega_{i}$ at $x_{i}$.

The radiance to the reflection direction $\omega_{o}$ at the surface point $x_{i}$ is expressed

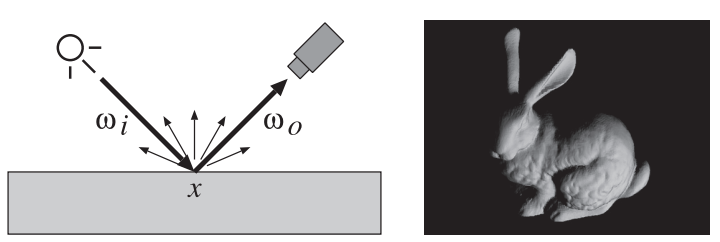

(a) Opaque object

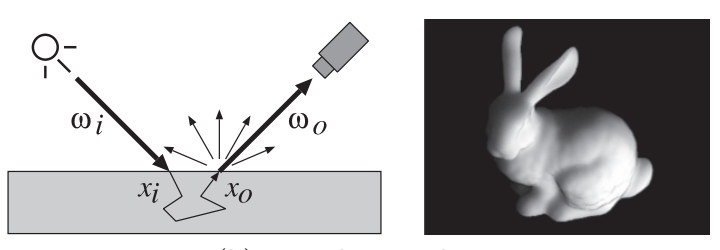

(b) Translucent object

Fig. 2 The difference between BRDF and BSSRDF. 
130 Analysis of Subsurface Scattering Based on Dipole Approximation

by

$$
L_{o}\left(x_{o}, \omega_{o}\right)=\int_{A} \int_{\Omega^{+}} S\left(x_{i}, \omega_{i}, x_{o}, \omega_{o}\right) L_{i}\left(x_{i}, \omega_{i}\right)\left(N \cdot \omega_{i}\right) d \omega_{i} d x_{i}
$$

Here, $L_{i}\left(x_{i}, \omega_{i}\right)$ represents the power of the incident illumination from the direction $\omega_{i}$ at the surface point $x_{i} . A, \Omega^{+}$, and $N$ are the surface area, positive hemisphere, and surface normal at point $x_{i}$, respectively.

\subsection{Dipole Approximation}

In the computer graphics field, the photon mapping algorithm ${ }^{6)}$ is often used to express subsurface scattering. However, it requires huge computational cost to render realistic images. Jensen, et al. ${ }^{7)}$ proposed the dipole approximation to simulate subsurface light transport. It can render realistic images in a short time. Hence, we also use the dipole approximation to analyze translucent objects.

The dipole approximation assumes that the subsurface scattering does not depend on the incident and radiative directions. Hence, the BSSRDF $S\left(x_{i}, \omega_{i}, x_{o}, \omega_{o}\right)$ can be decomposed as

$$
S\left(x_{i}, \omega_{i}, x_{o}, \omega_{o}\right)=\frac{1}{\pi} F_{t, o}\left(\eta, \omega_{o}\right) R\left(x_{i}, x_{o}\right) F_{t, i}\left(\eta, \omega_{i}\right) .
$$

Here, $F_{t}(\eta, \omega)$ is the Fresnel function when the ray transmits from the direction $\omega$ to the boundary whose relative index of refraction is $\eta$.

$R\left(x_{i}, x_{o}\right)$ is the diffuse subsurface reflectance of light entering at point $x_{i}$ and exiting at point $x_{o}$. The $R\left(x_{i}, x_{o}\right)$ is approximated to $R(d)$ as a function of the distance $d=\left\|x_{o}-x_{i}\right\|$ as follows,

$$
R(d)=\frac{\alpha}{4 \pi}\left\{z_{r}\left(\sigma_{t r}+\frac{1}{d_{r}}\right) \frac{e^{-\sigma_{t r} d_{r}}}{d_{r}^{2}}+z_{v}\left(\sigma_{t r}+\frac{1}{d_{v}}\right) \frac{e^{-\sigma_{t r} d_{v}}}{d_{v}^{2}}\right\}
$$

Each variable is defined as follows.

$$
\begin{aligned}
& d_{r}=\sqrt{d^{2}+z_{r}^{2}}, \quad d_{v}=\sqrt{d^{2}+z_{v}^{2}}, \quad z_{r}=\frac{1}{\sigma_{t}^{\prime}}, \quad z_{v}=z_{r}\left(1+\frac{4}{3} A\right) \\
& A=\frac{1+F_{d r}}{1-F_{d r}}, \quad F_{d r}=-\frac{1.440}{\eta^{2}}+\frac{0.710}{\eta}+0.668+0.0636 \eta \\
& \sigma_{t r}=\sqrt{3 \sigma_{a} \sigma_{t}^{\prime}}, \quad \sigma_{t}^{\prime}=\sigma_{s}^{\prime}+\sigma_{a}, \quad \sigma_{s}^{\prime}=\sigma_{s}(1-g), \quad \alpha=\frac{\sigma_{s}^{\prime}}{\sigma_{t}^{\prime}}
\end{aligned}
$$

The scattering coefficient $\sigma_{s}$, reduced scattering coefficient $\sigma_{s}^{\prime}$ and absorption coefficient $\sigma_{a}$ are inherent parameters of the material. $g$ is the mean cosine of the

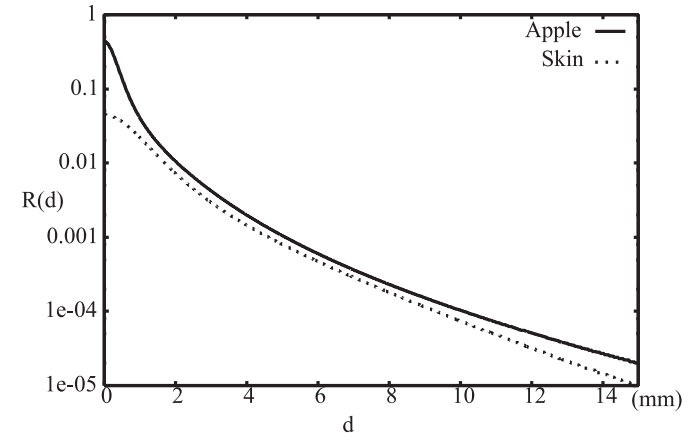

Fig. 3 Examples of $R\left(d\right.$ ) (Apple: $\sigma_{s}^{\prime}=2.29, \sigma_{a}=0.003, \eta=1.3$. Skin: $\sigma_{s}^{\prime}=0.74$, $\sigma_{a}=0.032, \eta=1.3$

scattering angle, which is zero when the scattering is isotropic. Hence, subsurface scattering is defined by the three parameters, $\sigma_{s}^{\prime}, \sigma_{a}$, and $\eta$, if we assume isotropic scattering $(g=0)$.

Figure 3 shows how the diffuse subsurface reflectance $R(d)$ changes with varying parameters. This graph shows that the power of the scattering light attenuates with distance from the incident point when the parameters corresponding to apple and skin ${ }^{7)}$ are set.

\section{Analysis of Subsurface Scattering}

\subsection{Conditions}

Our method estimates the parameters for the dipole approximation from only a single image taken in a known arbitrary illumination environment. First, we clarify the conditions for the proposed analysis method.

Geometry: The 3-D location of the camera and the 3-D shape of the target object are known.

Material: The material is homogeneous and scattering is isotropic.

Illumination: The distribution of the illumination at each surface point $L_{i}\left(x_{i}, \omega_{i}\right)$ is known.

\subsection{Linear Formulation}

The object surface is divided into $m$ small patches and the normal vector $N$ is calculated for each patch. The radiance $L_{o}\left(P_{j}\right)$ of patch $P_{j}$ is expressed by 
131 Analysis of Subsurface Scattering Based on Dipole Approximation

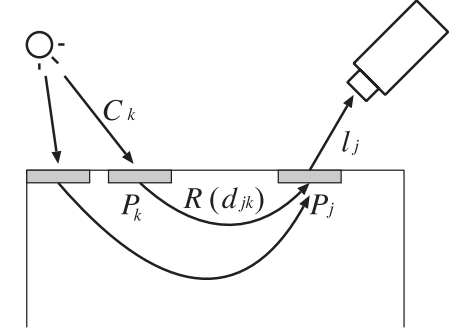

Fig. 4 Formulation of radiance.

$$
\begin{aligned}
L_{o}\left(P_{j}\right) & =\frac{1}{\pi} F_{t, o}\left(\eta, \omega_{o}, N_{j}\right) \\
& \sum_{k=1}^{m}\left\{R\left(d_{j k}\right) \int_{\Omega^{+}} L_{i}\left(P_{k}, \omega_{i}\right) F_{t, i}\left(\eta, \omega_{i}, N_{k}\right) \max \left(0, N_{k} \cdot \omega_{i}\right) d \omega_{i}\right\}
\end{aligned}
$$

Here, $d_{j k}$ denotes the length between $P_{j}$ and $P_{k}$, and $L_{i}\left(P_{k}, \omega_{i}\right)$ denotes irradiance from the direction $\omega_{i}$ at $P_{k}$. We set $\eta=1.3$ based on the research of Jensen et al. ${ }^{7)}$ and Goesele, et al. ${ }^{14)}$. Hence, $F_{t, o}\left(\eta, \omega_{o}, N_{j}\right)$ and $F_{t, i}\left(\eta, \omega_{i}, N_{k}\right)$ are calculated in advance from the 3 -D shape, the illumination, and the 3 -D position of the camera.

Now, we set $l_{j}$ and $c_{k}$.

$$
\begin{aligned}
& l_{j}=\pi L_{o}\left(P_{j}\right) / F_{t, o}\left(\eta, \omega_{o}, N_{j}\right), \\
& c_{k}=\int_{\Omega^{+}} L_{i}\left(P_{k}, \omega_{i}\right) F_{t, i}\left(\eta, \omega_{i}, N_{k}\right) \max \left(0, N_{k} \cdot \omega_{i}\right) d \omega_{i} .
\end{aligned}
$$

Then, $l_{j}$ is represented by

$$
l_{j}=\sum_{k=1}^{m}\left(R\left(d_{j k}\right) c_{k}\right),
$$

as shown in Fig. 4. By using $\mathbf{l}, \mathbf{c}$ and $\mathbf{R}$ defined below,

$$
\mathbf{l}=\left[l_{1}, l_{2}, \ldots, l_{m}\right]^{\mathrm{T}}, \quad \mathbf{c}=\left[c_{1}, c_{2}, \ldots, c_{m}\right]^{\mathrm{T}},
$$

$$
\mathbf{R}=\left[\begin{array}{ccccc}
R\left(d_{11}\right) & \ldots & R\left(d_{1 k}\right) & \ldots & R\left(d_{1 m}\right) \\
\vdots & \ddots & \vdots & & \vdots \\
R\left(d_{j 1}\right) & \ldots & R\left(d_{j k}\right) & \ldots & R\left(d_{j m}\right) \\
\vdots & & \vdots & \ddots & \vdots \\
R\left(d_{m 1}\right) & \ldots & R\left(d_{m k}\right) & \ldots & R\left(d_{m m}\right)
\end{array}\right]
$$

the subsurface scattering can be expressed by the following linear formulation,

$$
\mathbf{l}=\mathbf{R c} \text {. }
$$

The $\mathbf{l}$ and $\mathbf{c}$ are known, and the unknown $\sigma_{a}$ and $\sigma_{s}^{\prime}$ are included in $\mathbf{R}$. In principle, it is possible to estimate directly the best parameter set for $\sigma_{a}$ and $\sigma_{s}^{\prime}$ that minimizes $|\mathbf{l}-\mathbf{R c}|$ by varying the parameters. However, this direct approach is impractical owing to the huge computational time required. Multiplication of $\mathbf{R}$ and $\mathbf{c}$ corresponds to image rendering, and this often requires a few minutes depending on the number of patches. Hence, $\mathbf{R}$ is calculated first, and thereafter, the best parameter set for $\sigma_{a}$ and $\sigma_{s}^{\prime}$ is estimated.

The unknown $\mathbf{R}$ cannot be calculated from Eq. (13) because $m^{2}$ unknowns are included in $\mathbf{R}$, while only $m$ constraints are derived from Eq. (13). The actual value of $m$ is 3,750 in the experiment with a simulated image described in Section 4.1. This means that there are 14,062,500 unknowns and only 3,750 constraints. In the experiment with real images described in Section 4.2, the actual value of $m$ are 13,467 and 6,634 for a cube and pyramid, respectively. It is thus obvious that the number of unknowns needs to be reduced.

\subsection{Quantization of Distance between Patches}

To calculate $\mathbf{R}$ from $\mathbf{l}$ and $\mathbf{c}$, we propose a linear solution. The key idea is a piecewise-linear approximation of the diffuse subsurface reflectance $R(d)$. For the approximation, the continuous distances between the patches are quantized to discrete distances. This quantization enables us to reduce the number of unknowns. Based on the quantization, the best solution that minimizes the RMS error can be calculated using the Least Square method.

First, the distances $d_{11}, \ldots, d_{m m}$ are approximated by $n$ quantized distances $d_{1}^{\prime}, d_{2}^{\prime}, \ldots, d_{n}^{\prime} . R_{i}^{\prime}$ denotes the diffuse subsurface reflectance corresponding to the quantized distance $d_{i}^{\prime}$. The $d_{i}^{\prime}$ satisfy the following conditions.

$$
d_{1}^{\prime}=0, \quad d_{n}^{\prime}>\max \left(d_{j k}\right),
$$


132 Analysis of Subsurface Scattering Based on Dipole Approximation

$$
d_{1}^{\prime}<d_{2}^{\prime}<\cdots<d_{n}^{\prime} .
$$

$d_{j k}$ is quantized by finding $i$ that satisfies

$$
d_{i}^{\prime} \leq d_{j k}<d_{i+1}^{\prime}
$$

and defined by

$$
d_{j k}=\beta_{j k} d_{i}^{\prime}+\left(1-\beta_{j k}\right) d_{i+1}^{\prime}, \quad \beta_{j k}=\frac{d_{i+1}^{\prime}-d_{j k}}{d_{i+1}^{\prime}-d_{i}^{\prime}} .
$$

Then, the diffuse subsurface reflectance $R\left(d_{j k}\right)$ is approximated by linear interpolation as follows.

$$
R\left(d_{j k}\right) \simeq \beta_{j k} R_{i}^{\prime}+\left(1-\beta_{j k}\right) R_{i+1}^{\prime} .
$$

Using $n$ dimensional vectors

$$
\begin{aligned}
& \mathbf{w}_{\mathbf{j k}}=\left[\begin{array}{llllll}
\cdots & 0 & \beta_{j k} & \left(1-\beta_{j k}\right) & 0 & \cdots
\end{array}\right], \\
& \mathbf{r}=\left[\begin{array}{llll}
R_{1}^{\prime} & R_{2}^{\prime} & \ldots & R_{n}^{\prime}
\end{array}\right]^{\mathrm{T}}
\end{aligned}
$$

Equation (18) is represented by

$$
R\left(d_{j k}\right) \simeq \mathbf{w}_{\mathbf{j k}} \mathbf{r}
$$

From Eqs. (10) and (21), $l_{j}$ is represented by

$$
l_{j} \simeq \sum_{k=1}^{m}\left(c_{k} \mathbf{w}_{\mathbf{j k}} \mathbf{r}\right)
$$

Hence, Eq. (13) is approximated as

$$
\mathbf{l} \simeq \mathbf{W r}, \quad \mathbf{W}=\left[\begin{array}{c}
\sum_{k=1}^{m}\left(c_{k} \mathbf{w}_{\mathbf{1 k}}\right) \\
\sum_{k=1}^{m}\left(c_{k} \mathbf{w}_{\mathbf{2 k}}\right) \\
\vdots \\
\sum_{k=1}^{m}\left(c_{k} \mathbf{w}_{\mathbf{m k}}\right)
\end{array}\right] .
$$

This equation includes $n$ unknowns and $m$ constraints. If $n \leq m, \mathbf{r}$ can be calculated linearly as

$$
\mathbf{r}=\mathbf{W}^{+} \mathbf{l} \text {. }
$$

Here, $\mathbf{W}^{+}$is the pseudo inverse of $\mathbf{W}$. In practice, the radiance of all patches is not fully recorded in one input image. Hence, only the rows corresponding to visible patches are extracted from $\mathbf{l}$ and $\mathbf{W}$, then $\mathbf{r}$ is calculated from the partial $\mathbf{l}$ and $\mathbf{W}$.

Since the diffuse subsurface reflectance $R(d)$ is smooth and a monotonically decreasing function as shown in Fig. 3, the quantization does not cause crucial errors if the quantization step is small.

Since the actual value of $n$ depends on the quantization step, it varies between 50 and 450 in the experiment with a simulated image described in Section 4.1. In the experiment with real images described in Section 4.2 , the actual value of $n$ is 10 . Hence, the problem is over-constrained.

\subsection{Parameter Estimation}

Once the diffuse subsurface reflectance $R_{i}^{\prime}$ has been calculated for each distance $d_{i}^{\prime}$, the dipole approximation is fit to the data. The diffuse subsurface reflectance $R(d)$ is uniquely decided by only two parameters $\sigma_{s}^{\prime}$ and $\sigma_{a}$, since we assume that the refractive index $\eta$ is known. The optimal two parameters are estimated from

$$
\arg \min _{\sigma_{s}^{\prime}, \sigma_{a}} \sum_{i=1}^{n}\left(R_{i}^{\prime}-R\left(d_{i}^{\prime}\right)\right)^{2}
$$

and the subsurface scattering model for the target material is obtained.

\section{Experiment}

\subsection{Simulated Image}

First, we evaluated how the quantization of distance affects the accuracy of the estimated parameters. For a more precise evaluation, a simulated scene was used, and the same subsurface scattering model was used for both rendering and analyzing to avoid other adverse effects. For rendering the simulated image, we originally implemented the renderer based on Jensen's algorithm ${ }^{7)}$. Figure 5 shows the illumination environment captured using a mirrored ball. Under this illumination, an image of a pyramid was rendered using dipole approximation $\left(\sigma_{s}^{\prime}=2.19, \sigma_{a}=0.002, \eta=1.3\right)$ as shown in Fig. 6 (a). There are 3,750 patches in this pyramid model.

To obtain the best parameter set for the dipole model, all possible combinations 
133 Analysis of Subsurface Scattering Based on Dipole Approximation

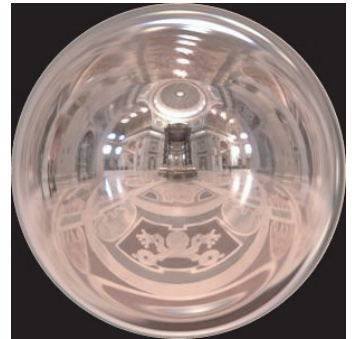

Fig. 5 Illumination environment (http://www.debevec.org/Probes/).

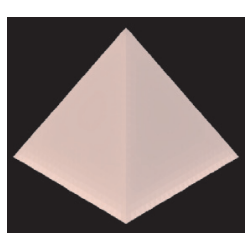

(a) input image

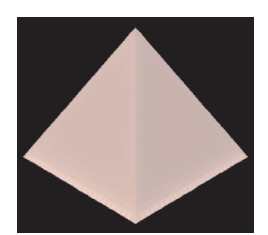

(b) resynthesized image
Fig. 6 Simulated image. (a) input image for given parameters $\left(\sigma_{s}^{\prime}=2.19, \sigma_{a}=0.002\right.$, and $\eta=1.3)$. (b) resynthesized image for the estimated parameters $\left(\sigma_{s}^{\prime}=2.34, \sigma_{a}=0.009\right)$.

Table 1 Range and step of the parameters.

\begin{tabular}{c|c|c}
\hline \hline & $\sigma_{s}^{\prime}$ & $\sigma_{a}$ \\
\hline minimum & 0.01 & 0.000 \\
maximum & 3.00 & 0.010 \\
step & 0.01 & 0.001 \\
\hline
\end{tabular}

of the parameters were tested to avoid local minima. The ranges and steps used for varying the parameters are given in Table 1. Figure 7 shows the fitting errors for all combinations of $\sigma_{s}^{\prime}$ and $\sigma_{a}$, with a quantization distance of $0.35 \mathrm{~mm}$. In this figure, the error values are expressed in color, while a red ' + ' marker indicates the estimated parameter set that minimizes the fitting error. We can see that an obvious global minimum exists for $\sigma_{s}^{\prime}$ and that the estimation of $\sigma_{s}^{\prime}$ is stable. On the other hand, even if $\sigma_{a}$ changes, the error does not vary while the distribution is smooth. In other words, the estimation of $\sigma_{a}$ tends to be unstable. In fact, $R(d)$ does not change dramatically as $\sigma_{a}$ varies. Figure 8

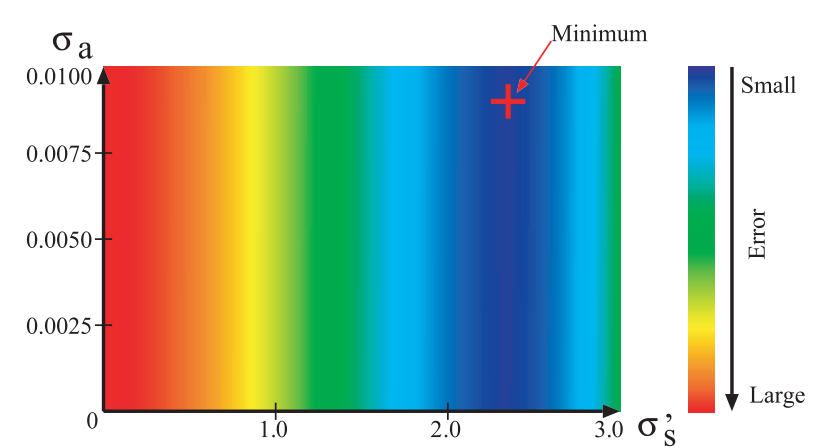

Fig. 7 Error distribution when quantizing distance is $0.35 \mathrm{~mm}$.

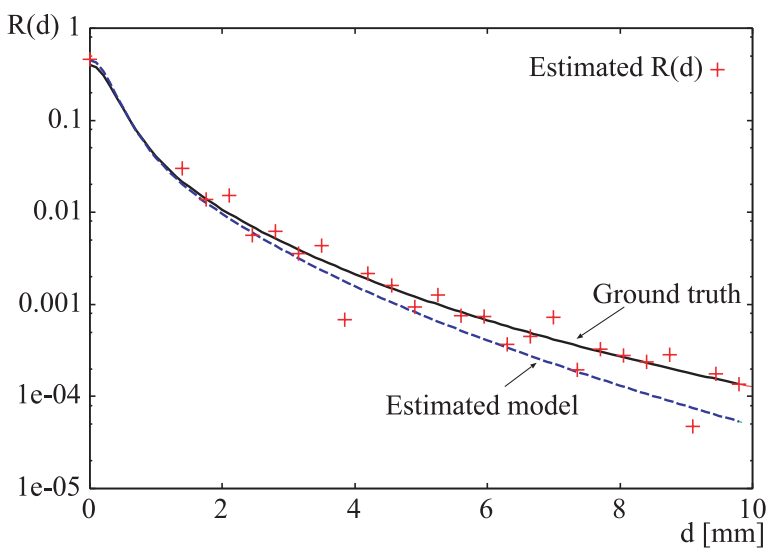

Fig. 8 Fitting result when quantizing distance is $0.35 \mathrm{~mm}$.

shows the relationship between the estimated diffuse subsurface reflectance $R(d)$ and the fitted dipole approximation with a quantization distance of $0.35 \mathrm{~mm}$. Since the estimated $R(d)$ include some outliers, the fitted dipole approximation slightly underestimates the ground-truth kernel for increasing the distance $d$.

Next, we evaluated the accuracy of the estimated parameters as the quantization distance varied between $0.05 \mathrm{~mm}$ and $0.50 \mathrm{~mm}$ with a $0.05 \mathrm{~mm}$ step. The parameters minimizing the fitting error were estimated for each quantization distance as listed in Table 2. While the estimated $\sigma_{s}^{\prime}$ is close to the ground truth, 
134 Analysis of Subsurface Scattering Based on Dipole Approximation

\begin{tabular}{c|c|c|c}
\multicolumn{5}{c}{ Table 2 Estimated parameters and PSNR. } \\
\hline \hline Sampling [mm] & $\sigma_{s}^{\prime}$ & $\sigma_{a}$ & PSNR [dB] \\
\hline 0.05 & 2.14 & 0.000 & 26.47 \\
0.10 & 2.20 & 0.007 & 42.12 \\
0.15 & 2.19 & 0.004 & 30.58 \\
0.20 & 2.19 & 0.009 & 33.47 \\
0.25 & 2.19 & 0.005 & 28.69 \\
0.30 & 2.32 & 0.009 & 29.78 \\
0.35 & 2.34 & 0.009 & 47.93 \\
0.40 & 2.22 & 0.009 & 25.76 \\
0.45 & 2.18 & 0.009 & 20.35 \\
0.50 & 2.40 & 0.009 & 23.43 \\
\hline Ground truth & 2.19 & 0.002 & - \\
\hline \multicolumn{4}{c}{}
\end{tabular}

$\sigma_{a}$ tends to be larger and unstable.

To evaluate the accuracy of the estimated parameters, images were resynthesized using the estimated parameters and these images were compared with the input image. For a quantitative comparison, the difference was measured by the PSNR (Peak Signal to Noise Ratio). The PSNR is often used to compute the difference between two images, where a large PSNR indicates that the two images are similar. The signal corresponds to the pixel value in the input image while the noise is the difference in the pixel values of the input and resynthesized images.

The PSNR for each quantizing distance is given in Table 2. It is generally stated that we cannot distinguish two images if the PSNR is greater than $40 \mathrm{~dB}$. The PSNRs are greater than $40 \mathrm{~dB}$ for distances of $0.10 \mathrm{~mm}$ and $0.35 \mathrm{~mm}$, and are around $30 \mathrm{~dB}$ between these distances as shown in Fig. 9. Figure 6 (b) shows the resynthesized image of the pyramid using the estimated parameters for distance of $0.35 \mathrm{~mm}$. As the PSNR is $47.93 \mathrm{~dB}$, we cannot see any differences from the input image in Fig. 6 (a).

If the quantizing distance is large, the quantizing error affects the parameter estimation. Inversely, too small a quantizing distance increases the number of unknowns, hence the calculation of the pseudo inverse in Eq. (24) becomes unstable. In fact, the PSNR is small for the short distance $0.05 \mathrm{~mm}$. Resolving the optimal quantizing distance is one of our future tasks.

Although all possible combinations of the parameters were tested to obtain

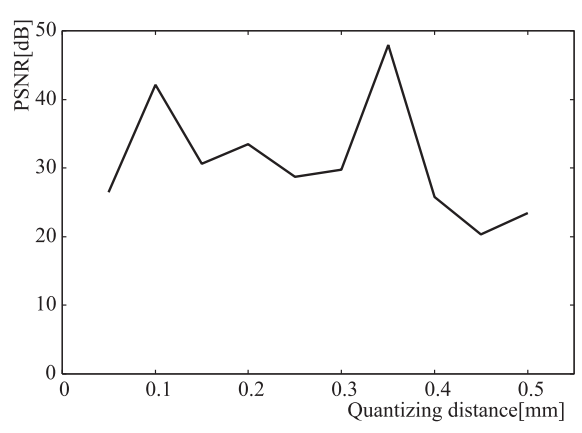

Fig. 9 Relationship between quantizing distance and PSNR.

the best parameter set, the total computation time is less than 1 second using a conventional Pentium4 PC. Of course, the computation time increases, if we set a smaller step for varying the parameters. In this case, a kind of steepest descent method may be effective in speeding up the computation since the error distribution is smooth and does not have local minima.

\subsection{Real Images}

Next, we estimated parameters for real images of objects made of various translucent materials. The purpose of this experiment was to evaluate stability when the assumed subsurface scattering model and actual phenomena are not the same. The images were captured with a Nikon D80 digital SLR camera using raw mode (12 bit). Despite the fact that our method is able to work under general illumination, we used a single white LED as illumination to allow us to measure the illumination more precisely. The 3-D positions of the camera and LED were measured by hand. To avoid surface reflection and single scattering, we used cross-polarization. In other words, linear polarizers were placed in front of the camera and light source, and each polarizer was manually rotated until no specular reflection was observed through the camera. The target objects were placed on a light absorbing black sheet in a dark room as shown in Fig. 10 (a).

Cubes and pyramids ${ }^{\star 1}$, made of three different materials namely polypropylene $(\mathrm{PP})$, polyethylene (PE), and polyoxymethylene (POM), were used as target

$\star 1$ The pyramid has a base that is required by its manufacture. 
135 Analysis of Subsurface Scattering Based on Dipole Approximation

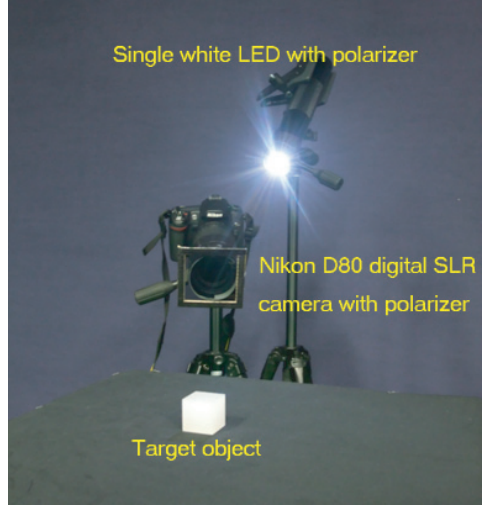

(a) equipment

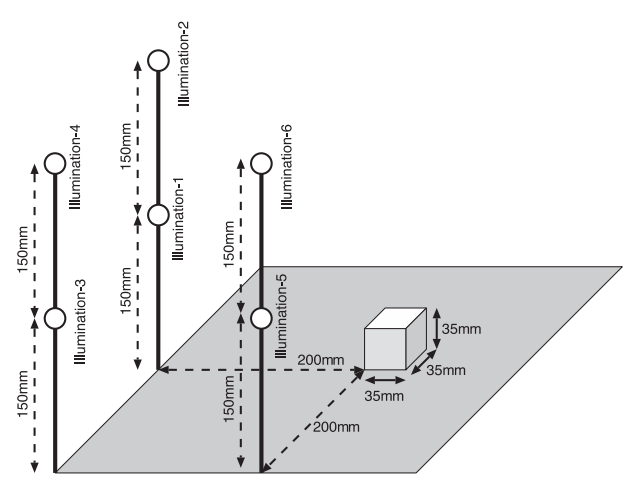

(b) location of the point light sources
Fig. 10 Setup for the actual experiment.

objects. There are, respectively, 13,467 and 6,634 patches on the cube and pyramid. Each object was captured under six different illumination conditions. The locations of the light source in relation to the target object are illustrated in Fig. 10 (b). Since the distance from the source to the object is relatively small, the incident angle varies for every patch. The irradiance also attenuates according to the distance between the source and the patches. Hence, the irradiance is not uniform over the surface of the target object. In total, 36 images (2 shapes $\times 3$ materials $\times 6$ illuminations) were captured. Figure 11 shows the images of the cubes and pyramids made of PP, PE, and POM under 'illumination-3'. The camera settings, such as shutter speed and exposure, are the same for all images.

In this experiment, the quantization distance was fixed at $1 \mathrm{~mm}$. The range and step used for varying the parameters were the same as in the simulated scene in Section 4.1. Figure 12 shows the fitting results for the cube illuminated from the position referred as 'illumination-3'. In the graph, ' + ' markers indicate the computed diffuse subsurface reflectances, while the lines denote the fitting results from the dipole approximation for each material. The estimated parameters $\sigma_{s}^{\prime}$ and $\sigma_{a}$ for all images are graphically illustrated in Fig. 13. The rendering of the Stanford bunnies using the averaged parameter set for each material are shown in Fig. 14
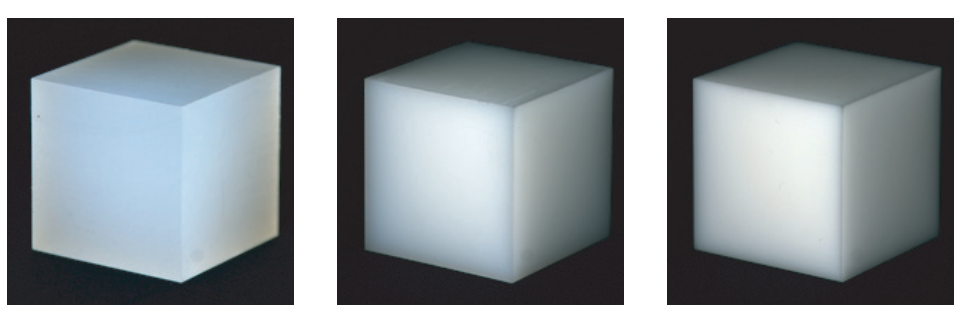

(a) Cube
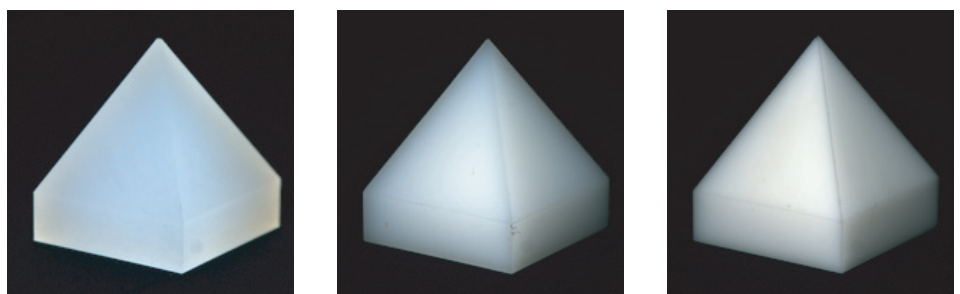

(b) Pyramid

Fig. 11 Real translucent objects (leftmost column: polypropylene (PP), center column: polyethylene (PE), rightmost column: polyoxymethylene (POM)).

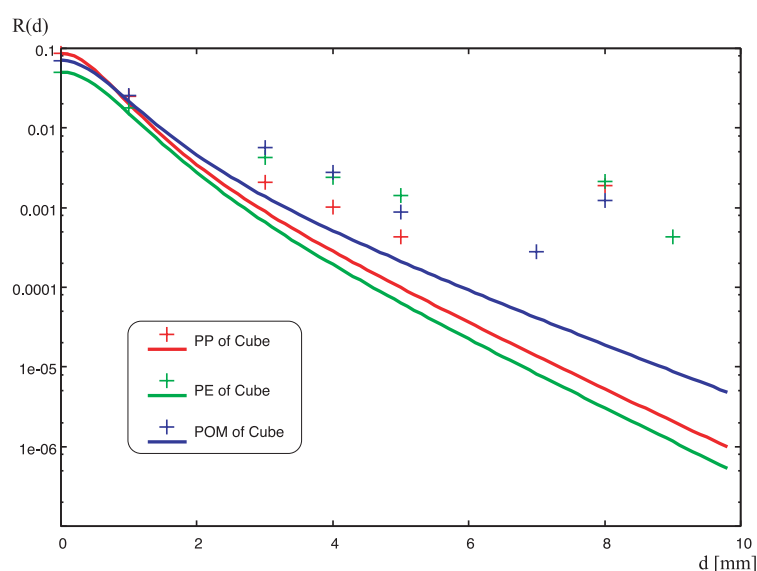

Fig. 12 Examples of fitting results for the cube. The ' + ' markers indicate the estimated diffuse subsurface reflectances, while the lines denote the fitting results using a dipole approximation. 


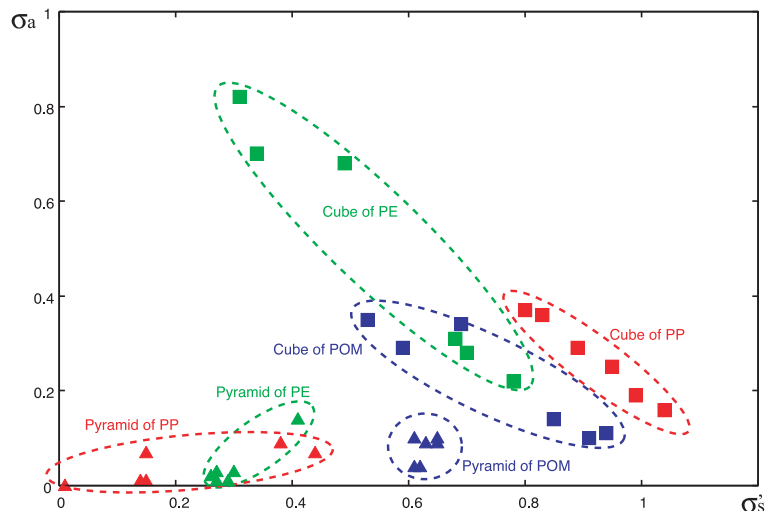

Fig. 13 Estimated parameters from the dipole approximation for all real images.

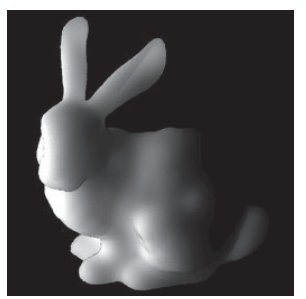

(a) PP

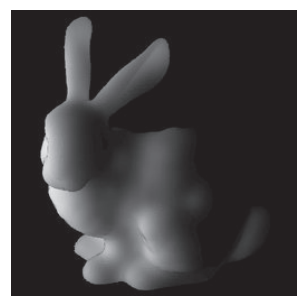

(b) PE

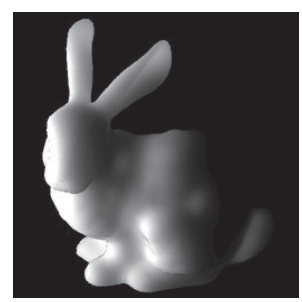

(c) POM
Fig. 14 Rendered Stanford bunnies.

Since the ground truth of the parameters was not known, we evaluated the estimated parameters by consistency. Ideally, the same parameters should be obtained for the same material even if the illumination or shape differs. In the experiment, similar parameters were estimated for the same shape and material The parameters for the different pyramids were not strongly affected by the differences in illumination. However, different parameters were estimated for the cube and pyramid. One of the possible reasons for this is that the dipole approximation assumes a semi-infinite slab, and thus cannot be used principally for analyzing and rendering complex shapes. The unstable result seems to be due to a mismatch between the scattering model and actual phenomena. Investigating an appropriate BSSRDF model that is suitable for image analysis of complex shaped objects is our future work.

\subsection{Discussion}

\subsubsection{Reasonability of the Dipole Approximation}

In this research, we used the dipole approximation for analyzing subsurface scattering. Since the BSSRDF is explicitly and mathematically given in the dipole approximation, the inverse problem that tries to find the BSSRDF from the radiance information can be solved easily compared with Monte Carlo ray tracing or photon mapping. However, a mismatch between the BSSRDF model and the actual phenomena caused unstable results. The dipole approximation originates from optical physics, and is designed for a semi-infinite material with a planar surface.

In fact, good results were obtained for the simulated image because the same model was used for rendering. However, the same accuracy cannot be expected for real images since these complex shaped objects do not fit the design of the dipole approximation.

Consequently, we need to develop a new BSSRDF model specifically for analyzing complex shaped objects. The data-driven approach ${ }^{17)}$ that records actual light transport is one of the potential candidates for handling more generic scattering behaviors.

\subsubsection{Radiometric Calibration}

Since we did not perform radiometric calibration in our experiments, we do not know the physical unit of a pixel value. Therefore we empirically rely on the 'GAIN' constant to convert the radiances to pixel values. To ascertain the absolute scale of the intensities and the ratio between the scattering and absorption parameters, we need to improve on this simple method.

\subsubsection{Adaptive Sampling of the Distances}

In our implementation, we set the quantization distance at even intervals. This approach may be inadequate for rapidly changing ranges and redundant for nonvarying ranges. The optimal distances for quantization depend on the 3 -D shape of the object. Adaptive sampling will be investigated in a future work to realize a better piecewise-linear approximation of the diffuse subsurface reflectance. 


\subsubsection{Relative Index of Refraction}

In our method, we set the relative index of refraction $\eta$ to 1.3 without any plausible reason. This decision has no foundation. If we estimate the value of $\eta$ together with the parameters for the dipole approximation, the problem becomes much more difficult. Automatically assigning a value to $\eta$ is another of our future works.

\subsubsection{Ground Truth}

The main reason for not being able to present objective evaluations in the experiment using real images, is that we do not know the ground truth of the parameters for PP, PE, and POM. In this respect, Tsumura, et al. ${ }^{13)}$ used phantoms with known physical properties to validate their reconstruction. It remains a future task to construct such phantoms and evaluate the estimated parameters.

\subsubsection{Illumination in a Real Environment}

Our method can be used in any illumination environment in which the illumination is known. Measuring the illumination environment is, however, a difficult task. Images with high dynamic ranges need to be captured precisely because general illumination includes both strong light sources and weak inter-reflections. Depending on the scene, the 3-D locations of all light sources must be recorded. Furthermore, the cross-polarization technique is difficult to apply. Measuring the illumination environment is another of our future tasks.

\section{Conclusion}

We proposed a new method to analyze subsurface scattering from a single image taken in a known arbitrary illumination environment. We showed that diffuse subsurface reflectance can be calculated linearly by quantizing the distances between each pair of surface points. We provided a solution to analyze subsurface scattering by inversely tracing the image rendering process, while previous approaches attempted to measure the subsurface scattering directly using special lighting devices such as a laser beam or projector.

Although accurate parameters could be estimated in the simulated experiment using an appropriate quantization distance, problems of stability and accuracy were still apparent since different parameters were estimated for the same mate- rials in the experiments with real objects. Although the analysis is not stable, we believe that our method is a significant first step in analyzing the appearance of translucent objects in a known arbitrary illumination environment. Our future work is to enhance the analyzing algorithm by using more suitable BSSRDF models. Detailed verification using a more complex scene is also necessary.

\section{References}

1) Nayar, S.K., Krishnan, G., Grossberg, M.D. and Raskar, R.: Fast Separation of Direct and Global Components of a Scene using High Frequency Illumination, Proc. SIGGRAPH2006, pp.935-944 (2006).

2) Hanrahan, P. and Krueger, W.: Reflection from layered surfaces due to subsurface scattering, Proc. SIGGRAPH1993, pp.165-174 (1993).

3) Stam, J.: Multiple scattering as a diffusion process, Proc. Eurographics Rendering Workshop (1995)

4) Dorsey, J., Edelman, A., Jensen, H.W., Legakis, J. and Pedersen, H.K.: Modeling and Rendering of Weathered Stone, Proc. SIGGRAPH'99, pp.225-234 (1999).

5) Pharr, M. and Hanrahan, P.: Monte Carlo evaluation of non-linear scattering equations for subsurface reflection, Proc. SIGGRAPH2000, pp.75-84 (2000).

6) Jensen, H.W. and Christensen, P.H.: Efficient Simulation of Light Transport in Scenes with Participating Media using Photon Maps, Proc. SIGGRAPH'98, pp.311320 (1998).

7) Jensen, H.W., Marschner, S.R., Levoy, M. and Hanrahan, P.: A Practical Model for Subsurface Light Transport, Proc. SIGGRAPH2001, pp.511-518 (2001).

8) Jensen, H.W. and Buhler, J.: A Rapid Hierarchical Rendering Technique for Translucent Materials, Proc. SIGGRAPH2002, pp.576-581 (2002).

9) Donner, C. and Jensen, H.W.: Light Diffusion in Multi-Layered Translucent Materials, Proc. SIGGRAPH2005, pp.1032-1039 (2005).

10) Ntziachristos, V., Yodh, A.G., Schnall, M. and Chance, B.: Concurrent MRI and diffuse optical tomography of breast after indocyanine green enhancement, Proc. National Academy of Science, Vol.97, No.6, pp.2767-2772 (2000).

11) Nitta, K., Matoba, O. and Yoshimura, T.: Characteristics of Weight Function in a Steady-state Diffusion Optical Tomography, IEEJ Trans. FM, Vol.127, No.7, pp.397-401 (2007).

12) Tsumura, N., Ojima, N., Sato, K., Shiraishi, M., Shimizu, H., Nabeshima, H. Akazaki, S., Hori, K. and Miyake, Y.: Image-based skin color and texture analysis/synthesis by extracting hemoglobin and melanin information in the skin, Proc. SIGGRAPH2003, pp.770-779 (2003).

13) Tsumura, N., Usuba, R., Takase, K., Nakaguchi, T., Ojima, N., Komeda, N. and Miyake, Y.: Image-based control of skin translucency, Proc. CGIV2006, pp.8-11 
(2006).

14) Goesele, M., Lensch, H.P.A., Lang, J., Fuchs, C. and Seidel, H.P.: Disco - Acquisition of Translucent Objects, Proc. SIGGRAPH2004, pp.835-844 (2004).

15) Fuchs, C., Goesele, M., Chen, T. and Seidel, H.P.: An Empirical Model for Heterogeneous Translucent Objects, Research Report MPI-I-2005-4-006 (2005).

16) Tariq, S., Gardner, A., Llamas, I., Jones, A., Debevec, P. and Turk, G.: Efficient Estimation of Spatially Varying Subsurface Scattering Parameters, Vision, Model ing, and Visualization (VMV2006) (2006).

17) Peers, P., vom Berge, K., Matusik, W., Ramamoorthi, R., Lawrence, J., Rusunkiewicz, S. and Dutré, P.: Compact Factored Representation of Heterogeneous Subsurface Scattering, Proc. SIGGRAPH2006, pp.746-753 (2006).

18) Weyrich, T., Matusik, W., Pfister, H., Bickel, B., Donner, C., Tu, C., McAndless, J., Lee, J., Ngan, A., Jensen, H.W. and Gross, M.: Analysis of Human Faces using a Measurement-Based Skin Reflectance Model, Proc. SIGGRAPH2006, pp.1013-1024 (2006).

19) Ghosh, A., Hawkins, T., Peers, P., Frederiksen, S. and Debevec, P.: Practical Modeling and Acquisition of Layered Facial Reflectance, Proc. SIGGRAPH Asia 2008.

20) Donner, C., Weyrich, T., d'Eon, E., Ramamoorthi, R. and Rusinkiewicz, S.: A Layered, Heterogeneous Reflectance Model for Acquiring and Rendering Human Skin, Proc. SIGGRAPH Asia 2008.

(Received September 23, 2008)

(Accepted June 1, 2009

(Released September 24, 2009)

(Communicated by Hiroshi Kawasaki)

Yasuhiro Mukaigawa received his M.E. and Ph.D. degrees

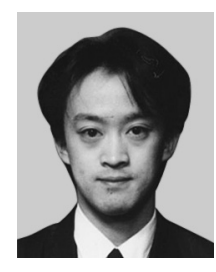

from University of Tsukuba in 1994 and 1997, respectively. He became a research associate at Okayama University in 1997, an assistant professor at University of Tsukuba in 2003, and an associate professor at Osaka University in 2004. His current research interests include photometric analysis and computational photography. He was awarded the MIRU Nagao Award in 2008. He is a member of IEICE, VRSJ, and IEEE.

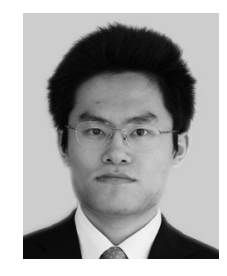

Kazuya Suzuki received his M.E. degree from Osaka University in 2008. He is currently working for Avantec Co., Ltd.

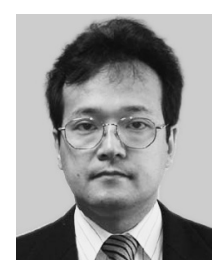

Yasushi Yagi is the Professor of Intelligent Media and Computer Science, and the Assistant Director of the Institute of Scientific and Industrial Research, Osaka university, Ibaraki, Japan. He received his Ph.D. degree from Osaka University in 1991. In 1985, he joined the Product Development Laboratory, Mitsubishi Electric Corporation, where he worked on robotics and inspections. He became a research associate in 1990, a lecturer in 1993 an associate professor in 1996, and a professor in 2003 at Osaka University. The international conferences for which he served as chair include: ROBIO2006 (Program co-chair), ACCV2007 (Program chair) and ACCV2009 (General chair). He is the editor of IEEE ICRA Conference Editorial Board (2007, 2008, 2009), the editor in chief of IPSJ Transactions on CVIM, and the associate editor in chief of IPSJ Transactions on CVA. He was awarded ACM VRST2003 Honorable Mention Award, IEEE ROBIO2006 Finalist of T.J. Tan Best Paper in Robotics, IEEE ICRA2008 Finalist for Best Vision Paper, and MIRU2008 Nagao Award. His research interests are computer vision, medical engineering and robotics. He is a member of IEICE, RSJ, and IEEE. 